\title{
Quantifying Elastic and Inelastic Electron Irradiation Damage in Transmission Electron Microscopy of 2D Materials
}

\author{
Toma Susi $^{1 *}$, Tibor Lehnert ${ }^{2}$, Ute Kaiser ${ }^{2}$, Jannik Meyer ${ }^{3}$, and Jani Kotakoski ${ }^{1}$ \\ 1. University of Vienna, Faculty of Physics, Vienna, Austria \\ 2. University of Ulm, Central Facility of Electron Microscopy, Ulm, Germany \\ 3. University of Tübingen, Institute of Applied Physics, Tübingen, Germany \\ * Corresponding author: toma.susi@univie.ac.at
}

Advances in transmission electron microscopy capabilities have made it an unparalleled tool for materials characterization down to their atomic structure and chemical bonding. Concurrently, the available two-dimensional materials make for ideal samples where each individual atom or vacancy can be resolved. Very recent work has also revealed the possibility of a new application for focused electron irradiation: the precise manipulation individual covalently bound impurity atoms in graphene [1-3], single-walled carbon nanotubes [4], and even silicon [5].

Evaluating the possibilities for such uses requires a precise understanding of the interactions of electrons with energies down to $15 \mathrm{keV}$. I will review existing models and experiments on radiation damage in 2D materials and present new low-voltage data for $\mathrm{MoS}_{2}$. Purely elastic knock-on damage cross sections can in some cases be accurately predicted from first principles [6], with remaining questions related to the choice of the exchange-correlation functional and the role of in-plane vibrations. Accurate modeling of dynamics into arbitrary angles is still computationally prohibitively expensive, and faster techniques such as machine learning potentials may be needed to describe long timescales and larger systems.

However, electron irradiation damage in non-metallic specimens including $\mathrm{MoS}_{2}$ and $\mathrm{hBN}$ is either dominated or at least influenced by inelastic excitations, whose quantitative theoretical description remains difficult. It is not clear how well models proposed for damage in oxides describe processes at the atomic scale, and whether their parameters such as radiolysis efficiencies can be predicted from first principles. Further, even in materials that can be purely described by knock-on damage in their pristine state, especially graphene, beam-induced dynamics of their impurities show significant discrepancies compared to the best available models [7].

Since the inelastic scattering cross section increases at lower electron energies, it is not possible to avoid damage by reducing the acceleration voltage as in the case of knock-on damage. Inelastic processes that may need to be incorporated into our models include core and valence ionisation, the interplay of the lifetime of electronic and other excitations with the time between electron impacts, the possibility of the same electron causing multiple excitations, and distinguishing direct bond breaking via localized charging or radiolysis from the weakening of bonds followed by elastic knock-on damage [8].

Atomically resolved measurements of electron irradiation damage in materials with different dielectric properties [8,9] are starting to emerge at multiple and ever-lower electron energies (Figure 1). Twodimensional materials offer the best chance for providing the experimental data needed for further model development, which we hope can then be adapted to provide a more general quantitative understanding of any type of damage caused by electron irradiation. 
References:

[1] M Tripathi et al., Nano Letters 18 (2018), p. 5319.

[2] C Su et al., arXiv:1803.01369 (2019).

[3] O Dyck et al., Small 14 (2018), p. 1801771.

[4] K Mustonen et al., arXiv:1902.03972 (2019).

[5] B Hudak et al., ACS Nano 12 (2018), p. 5873.

[6] T Susi et al., Nature Communications 7 (2016), p. 13040.

[7] T Susi et al., 2D Materials 4 (2017), p. 042004.

[8] T Lehnert et al., Applied Physics Letters 110 (2017), p. 033106.

[9] O Cretu et al., Micron 72 (2015), p. 21.

[10] The work was funded by the European Research Council (ERC) Grant No. 756277-ATMEN.

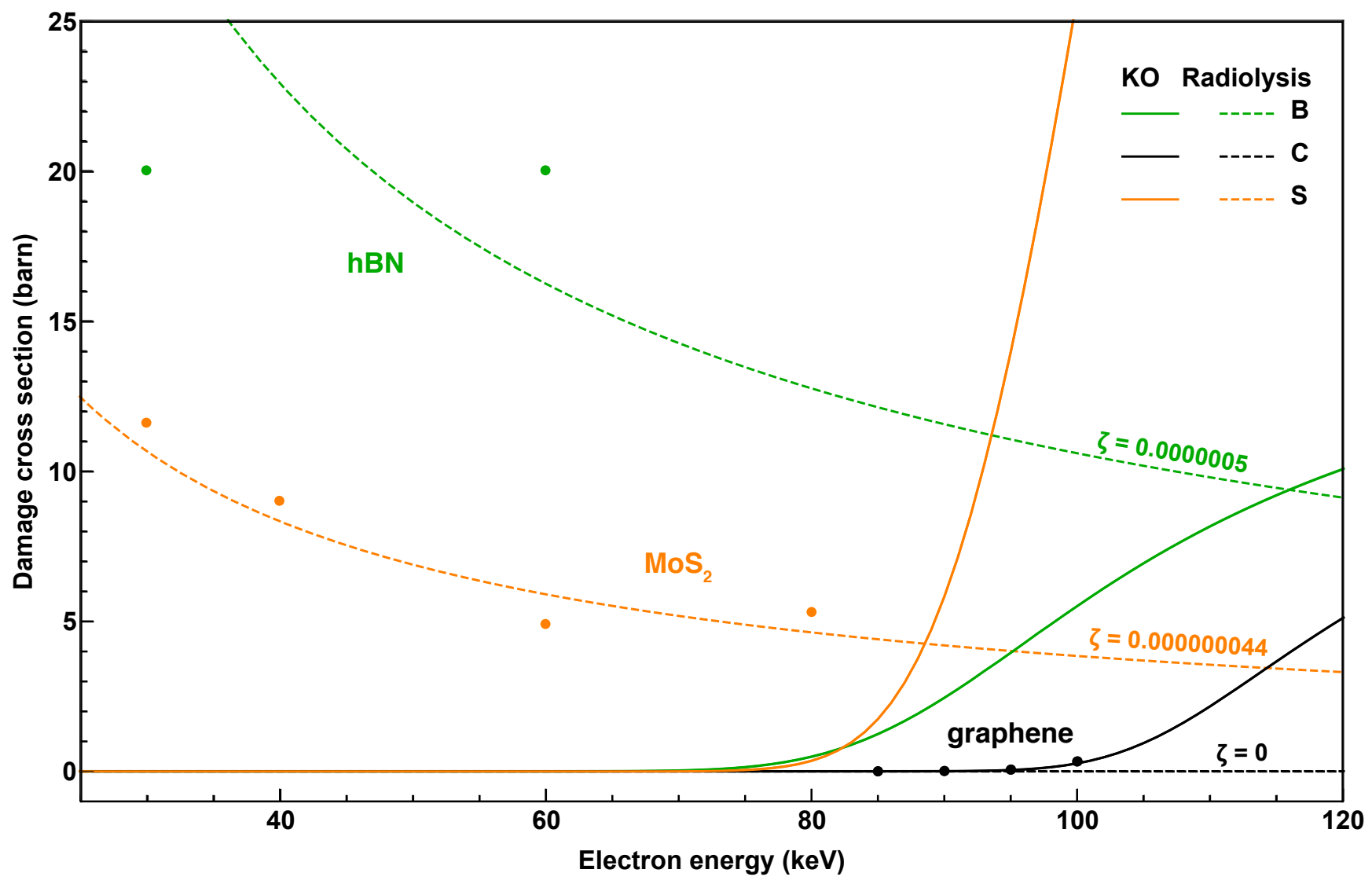

Figure 1. Electron irradiation damage cross sections from theory (lines) and atomically resolved measurements (points) for carbon atoms in graphene (black), sulfur atoms in $\mathrm{MoS}_{2}$ (orange), and boron atoms in $\mathrm{hBN}$ (green). The theoretical curves are for knock-on (KO) damage including the effect of vibrations [6] (solid lines) and for radiolysis (dashed lines; overlaid radiolysis efficiency parameter $\zeta$ is estimated to fit the data points). Experimental points for graphene are from Ref. [6], for hBN from Ref. [9], and for $\mathrm{MoS}_{2}$ from Ulm University (to be published). 